\title{
SYMMETRY OF ZEROS OF LERCH ZETA-FUNCTION FOR EQUAL PARAMETERS
}

\author{
RAMŪNAS GARUNKŠTIS AND ROKAS TAMOŠIŪNAS
}

\begin{abstract}
For most values of parameters $\lambda$ and $\alpha$, the zeros of the Lerch zeta-function $L(\lambda, \alpha, s)$ are distributed very chaotically. In this paper we consider the special case of equal parameters $L(\lambda, \lambda, s)$ and show by calculations that the nontrivial zeros either lie extremely close to the critical line $\sigma=1 / 2$ or are distributed almost symmetrically with respect to the critical line. We also investigate this phenomenon theoretically.
\end{abstract}

\section{INTRODUCTION}

Let $s=\sigma+i t$. Denote by $\{\lambda\}$ the fractional part of a real number $\lambda$. In this paper, $\varepsilon$ is any positive real number and $T$ always tends to plus infinity. In all theorems and lemmas, the numbers $\lambda$ and $\alpha$ are fixed constants.

For $0<\lambda, \alpha \leq 1$, the Lerch zeta-function is given by

$$
L(\lambda, \alpha, s)=\sum_{m=0}^{\infty} \frac{e^{2 \pi i \lambda m}}{(m+\alpha)^{s}} \quad(\sigma>1) .
$$

This function has analytic continuation to the whole complex plane except for a possible simple pole at $s=1$ (Lerch [16], Laurinčikas and Garunkštis [14]).

Let $l$ be a straight line in the complex plane $\mathbb{C}$, and denote by $\varrho(s, l)$ the distance of $s$ from $l$. Define, for $\delta>0$,

$$
L_{\delta}(l)=\{s \in \mathbb{C}: \varrho(s, l)<\delta\} .
$$

In Garunkštis and Laurinčikas [6], Garunkštis and Steuding [9], for $0<\lambda<1$ and $\lambda \neq 1 / 2$, it is proved that $L(\lambda, \alpha, s) \neq 0$ if $\sigma<-1$ and

$$
s \notin L_{\frac{\log 4}{\pi}}\left(\sigma=\frac{\pi t}{\log \frac{1-\lambda}{\lambda}}+1\right) .
$$

2010 Mathematics Subject Classification. Primary: 11M35; Secondary: 11M26.

Key words and phrases. Lerch zeta-function; nontrivial zeros; Speiser's equivalent for the Riemann hypothesis.

The first author is supported by grant No. MIP-049/2014 from the Research Council of Lithuania. 
For $\lambda=1 / 2,1$, from Spira [19] and [6] we see that $L(\lambda, \alpha, s) \neq 0$ if $\sigma<-1$ and $|t| \geq 1$. Moreover, in [6] it is showed that $L(\lambda, \alpha, s) \neq 0$ if $\sigma \geq 1+\alpha$. We say that a zero of $L(\lambda, \alpha, s)$ is nontrivial if it lies in the strip $-1 \leq \sigma<1+\alpha$ and we denote a nontrivial zero by $\rho=\beta+i \gamma$.

Let $\zeta(s)$ and $L(s, \chi)$ denote the Riemann zeta-function and the Dirichlet $L$-function accordingly. We have that

$$
L(1,1, s)=\zeta(s) \quad \text { and } \quad L(1 / 2,1 / 2, s)=2^{s} L(s, \chi)
$$

where $\chi$ is a Dirichlet character $\bmod 4$ with $\chi(3)=-1$. For these two cases, certain versions of the Riemann hypothesis $(\mathrm{RH})$ can be formulated. Similar cases are $L(1,1 / 2, s)=\left(2^{s}-1\right) \zeta(s)$ and $L(1 / 2,1, s)=$ $\left(1-2^{1-s}\right) \zeta(s)$. For all the other cases, it is expected that the real parts of zeros of the Lerch zeta-function form a dense subset of the interval $(1 / 2,1)$. This is proved for any $\lambda$ and transcendental $\alpha$ ([14, Theorem 4.7 in Chapter 8]).

In this paper, we investigate the zero distribution of the Lerch zetafunction $L(\lambda, \alpha, s)$ when the parameters are equal, i.e. $\lambda=\alpha$. The motivation for this are calculations which show that the first nontrivial zeros of $L(\lambda, \lambda, s)$ are often located almost on the critical line $\sigma=1 / 2$. Next are the first 4 zeros (rounded to two decimal numbers) of several Lerch zeta-functions.

$$
\begin{aligned}
& L(1 / 3,1 / 3, s): 0.50+3.99 i, 0.50+7.28 i, 0.50+9.54 i, 0.50+12.18 i . \\
& L(1 / 3,2 / 3, s): 0.86+5.68 i, 0.53+9.59 i, 0.86+12.66 i, 0.49+15.11 i . \\
& L(3 / 4,3 / 4, s): 0.50+9.69 i, 0.50+15.26 i, 0.50+18.65 i, 0.50+23.05 i . \\
& L(1 / 4,3 / 4, s): 1.03+5.24 i, 0.64+8.81 i, 0.76+11.96 i, 0.88+14.19 i .
\end{aligned}
$$

For a rational number $\lambda \neq 1 / 2,1$ it is expected that the function $L(\lambda, \lambda, s)$ has many zeros off the critical line. Our calculations then show that the zeros are almost symmetrically distributed with respect to the critical line. For example, for $L(3 / 4,3 / 4, s)$ we have the following zeros: $-0.10+120.60 i$ and $1+0.10+120.60 i ; 0.37+202.77 i$ and $1-0.37+202.77 i$. Usually such symmetry of zeros can be explained by the shape of the functional equation. A typical example is the Heillbronn Davenport zeta-function. Possibly such symmetry forces zeros to stay on the critical line more often. More on this see, for example, Bombieri and Hejhal [4], Balanzario and Sánchez-Ortiz [2], Garunkštis and Šimènas [10], Vaughan [22. For the Lerch zeta-function the following relation, usually called the functional equation, is true.

$$
\begin{aligned}
L(\lambda, \alpha, 1-s)= & (2 \pi)^{-s} \Gamma(s)\left(e^{\pi i \frac{s}{2}-2 \pi i \alpha \lambda} L(1-\alpha, \lambda, s)\right. \\
& \left.+e^{-2 \pi i \frac{s}{4}+2 \pi i \alpha(1-\{\lambda\})} L(\alpha, 1-\{\lambda\}, s)\right) .
\end{aligned}
$$


Various proofs of this functional equation can be found in Lerch [16], Apostol [1], Oberhettinger [18], Mikolás [17], Berndt [3], see also Lagarias and Li [11, 12. The Lerch zeta function has a second moment (Garunkštis, Laurinčikas, and Steuding [7]) and it is a universal function (Laurinčikas [13], Lee, Nakamura, Pańkowski [15]).

For $\lambda=\alpha$, we can rewrite (2) as

$$
\begin{aligned}
\overline{L(\lambda, \lambda, 1-\bar{s})}= & (2 \pi)^{-s} \Gamma(s) e^{-\pi i \frac{s}{2}+2 \pi i \lambda^{2}} L(\lambda, \lambda, s) \\
& +(2 \pi)^{-s} \Gamma(s) e^{\pi i \frac{s}{2}-2 \pi i(1-\lambda) \lambda} L(1-\lambda, 1-\{\lambda\}, s) \\
= & G(s) L(\lambda, \lambda, s)+P(s) .
\end{aligned}
$$

By the bound for the Lerch zeta-function and by Stirling's formula we see that, for any vertical strip, $|P(s)|<t^{A} e^{-\pi t}$ and $|G(s)| \geq t^{B}$ (see Lemma 6 and its proof below). Thus the shape of the formula (3) suggests that the nontrivial zeros of $L(\lambda, \lambda, s)$ should be distributed almost symmetrically with the respect of the critical line. However calculations in the next section show that this symmetry is not strict.

Denote by $N(\lambda, \alpha, T)$ the number of nontrivial zeros of the function $L(\lambda, \alpha, s)$ in the region $0<t<T$. For $0<\lambda, \alpha \leq 1$, we have $([6])$

$$
N(\lambda, \alpha, T)=\frac{T}{2 \pi} \log \frac{T}{2 \pi e \alpha \lambda}+O(\log T) .
$$

The next theorem shows that in the upper half-plane nontrivial zeros of the Lerch zeta-function with equal parameters on average are symmetrically distributed with a small error term.

Theorem 1. For $0<\lambda, \alpha \leq 1$,

$$
\sum_{0<\gamma \leq T}\left(\beta-\frac{1}{2}\right)=\frac{T}{4 \pi} \log \frac{\alpha}{\lambda}+O(\log T) .
$$

Now we consider the symmetry of the individual zeros. Let $\rho$ be a zero of $L(\lambda, \lambda, s)$. In view of (3) and Rouché's theorem we see that $L(\lambda, \lambda, s)$ has an almost symmetrical zero in some small disc $\mid s-(1-$ $\bar{\rho}) \mid<r$ if $P(s)$ is small and $L(\lambda, \lambda, s)$ is not very small on the edge of the disc. Thus we need a bound from below for $L(\lambda, \lambda, s)$ when $s$ is close to a zero.

Proposition 2. Let $0<\lambda, \alpha \leq 1$. Let $\sigma_{0} \in \mathbb{R}$ and $\Re s \geq \sigma_{0}$. Let $L(\lambda, \alpha, s) \neq 0$ and $d$ be the distance from $s$ to the nearest zero of $L(\lambda, \alpha, s)$. Then

$$
\frac{1}{|L(\lambda, \alpha, s)|}<\exp (C(|\log d|+1) \log t)
$$

where $C=C\left(\lambda, \alpha, \sigma_{0}\right)$ is a positive constant. 
The proposition will help us to prove the following theorem.

Theorem 3. Let $0<\lambda \leq 1$ and $A>0$ be such that $A C<\pi$, where $C=C(\lambda, \alpha,-1)$ is from Proposition 2. Let $\rho=\beta+i \gamma$ be a nontrivial zero of $L(\lambda, \lambda, s)$. If $\gamma$ is sufficiently large, then there is a radius $r$,

$$
\exp (-A \gamma / \log \gamma) \leq r \leq \exp (-A \gamma / \log \gamma) \log ^{2} \gamma
$$

such that the discs

$$
|s-\rho|<r \quad \text { and } \quad|s-(1-\bar{\rho})|<r
$$

contain the same number of zeros.

In the next section we present the computer calculations related to Theorem 3, Sections 3, 4, and 5 contain proofs of Theorem 1, Proposition 2, and Theorem 3 respectively.

\section{Computations}

This section is devoted to the more precise calculations of the first nontrivial zeros. If a nontrivial zero $\rho$ of $L(\lambda, \lambda, s)$ lies on the critical line, then by the functional equation (3) we have that $L(1-\lambda, 1-\lambda, \rho)=$ 0 . Similarly, if $L(\lambda, \lambda, s)$ has symmetrical zeros $\rho$ and $1-\bar{\rho}$ then again $L(1-\lambda, 1-\lambda, \rho)=0$. Let $\rho_{1}=0.50 \ldots+9.69 \ldots i, \rho_{2}=0.50 \ldots+15.26 \ldots i$, $\rho_{3}=0.50 \ldots+18.65 \ldots i, \rho_{4}=0.50 \ldots+23.05 \ldots i$ be the first four zeros of $L(3 / 4,3 / 4, s)$ indicated in the Introduction. We have that

$$
\begin{aligned}
& \left|L\left(1 / 4,1 / 4, \rho_{1}\right)\right|=2.73 \ldots, \\
& \left|L\left(1 / 4,1 / 4, \rho_{2}\right)\right|=0.13 \ldots, \\
& \left|L\left(1 / 4,1 / 4, \rho_{3}\right)\right|=0.48 \ldots, \\
& \left|L\left(1 / 4,1 / 4, \rho_{4}\right)\right|=1.15 \ldots
\end{aligned}
$$

Thus the zeros $\rho_{1}, \rho_{2}, \rho_{3}, \rho_{4}$ of $L(3 / 4,3 / 4, s)$ do not lie on the critical line. Using arbitrary-precision floating-point arithmetic computations, we get that

$$
\begin{aligned}
& \Re \rho_{1}=0.5+7.16 \ldots \cdot 10^{-14}, \\
& \Re \rho_{2}=0.5-6.08 \ldots \cdot 10^{-23}, \\
& \Re \rho_{3}=0.5-4.53 \ldots \cdot 10^{-27}, \\
& \Re \rho_{4}=0.5-1.11 \ldots \cdot 10^{-32} .
\end{aligned}
$$

The last four lines were computed in the following two ways: one by using findroot and the other by computing the contour integral which encloses only one zero $\rho$ of $L(3 / 4,3 / 4, s)$. For more details on computation methodology see the end of this section.

In the upper half-plane, the first pair of almost symmetrical zeros of $L(3 / 4,3 / 4, s)$ is $-0.10 \ldots+120.59 \ldots i$ and $1.10 \ldots+120.59 \ldots i$. These zeros are not strictly symmetrical, since

$$
|L(1 / 4,1 / 4,1.10 \ldots+120.59 \ldots i)|=3.94 \ldots \neq 0,
$$


TABLE 1. Distribution of the nontrivial zeros of $L(\lambda, \lambda, \sigma+i t)$ in $0<t<300 . N_{1}$ is the total number of the nontrivial zeros; $N_{2}$ is the number of the nontrivial zeros satisfying $|\Re \rho-1 / 2|>10^{-9}$, these zeros appear in almost symmetrical pairs; in the last column of the table, we have $100 N_{2} / N_{1}$.

\begin{tabular}{l|r|r|r}
$\lambda$ & $N_{1}$ & $N_{2}$ & $\%$ \\
\hline $1 / 2$ & 203 & 0 & 0.00 \\
$5 / 9$ & 193 & 28 & 14.51 \\
$4 / 7$ & 191 & 24 & 12.57 \\
$3 / 5$ & 186 & 14 & 7.53 \\
$5 / 8$ & 182 & 22 & 12.09 \\
$2 / 3$ & 176 & 18 & 10.23 \\
$7 / 10$ & 171 & 28 & 16.37 \\
$5 / 7$ & 169 & 30 & 17.75 \\
$3 / 4$ & 165 & 20 & 12.12 \\
$7 / 9$ & 161 & 26 & 16.15 \\
$4 / 5$ & 159 & 22 & 13.84 \\
$5 / 6$ & 155 & 22 & 14.19 \\
$6 / 7$ & 151 & 28 & 18.54 \\
$7 / 8$ & 150 & 30 & 20.00 \\
$8 / 9$ & 149 & 22 & 14.77 \\
$9 / 10$ & 147 & 24 & 16.33
\end{tabular}

$$
|L(1 / 4,1 / 4,-0.10 \ldots+120.59 \ldots i)|=23.49 \ldots \neq 0 .
$$

Further, we give a table (see Table 1) where the number of nontrivial zeros in $0<t<300$ is calculated for various cases of $L(\lambda, \lambda, s)$. For all those zeros, we have checked that $L(\lambda, \lambda, \rho)=0$ implies $L(1-\lambda, 1-\lambda, \rho) \neq$ 0 if $\lambda \neq \frac{1}{2}$. Thus, in Table 1, all zeros, except the case $\lambda=1 / 2$, are not strictly symmetrical with respect to the critical line.

Computations were validated with the help of Python with mpmath] package. We used the following expression of the Lerch zeta-function

\footnotetext{
${ }^{1}$ Fredrik Johansson and others. mpmath: a Python library for arbitrary-precision floating-point arithmetic (version 0.18), December 2013. http://mpmath.org/
} 
for rational parameters

$$
\begin{aligned}
L\left(s, \frac{b}{d}, \frac{b}{d}\right) & =\sum_{k=0}^{d-1} \sum_{m=0}^{\infty} \frac{\exp \left(2 \pi i \frac{b}{d}(d m+k)\right)}{\left(d m+k+\frac{b}{d}\right)^{s}} \\
& =d^{-s} \sum_{k=0}^{d-1} \exp \left(2 \pi i \frac{b}{d} k\right) \zeta\left(s, \frac{k d+b}{d^{2}}\right),
\end{aligned}
$$

where $\zeta(s, \alpha), 0<\alpha \leq 1$, is the Hurwitz zeta-function. The function $\zeta(s, \alpha)$ is implemented by the command zeta. Zero locations were calculated using findroot with Muller's method.

In this paper, all computer computations should be regarded as heuristic because their accuracy was not controlled explicitly.

\section{Proof of Theorem 1}

In [8], it was proved that, for $0<\lambda, \alpha \leq 1$,

$$
\sum_{|\gamma| \leq T}\left(\beta-\frac{1}{2}\right)=\frac{T}{2 \pi} \log \frac{\alpha}{\sqrt{\lambda(1-\{\lambda\})}}+O(\log T) .
$$

Theorem 1 can be derived from the proof of formula (6). Namely, from the proof of Theorem 1 in [8] we derive the following lemma.

Lemma 4. Let $b \geq 3$ be a constant. For $0<\lambda, \alpha \leq 1$,

$$
\sum_{0<\gamma \leq T}(b+\beta)=\left(b+\frac{1}{2}\right) \frac{T}{2 \pi} \log \frac{T}{2 \pi e \alpha \lambda}+\frac{T}{4 \pi} \log \frac{\alpha}{\lambda}+O(\log T) .
$$

Then the equality

$$
\sum_{0<\gamma \leq T}\left(\beta-\frac{1}{2}\right)=\sum_{0<\gamma \leq T}(b+\beta)-\left(b+\frac{1}{2}\right) \sum_{0<\gamma \leq T} 1
$$

together with the zero counting formula (4) gives Theorem 1 .

\section{Proof of Proposition 2}

We start from the following lemma.

Lemma 5. If $f(s)$ is regular, and

$$
\left|\frac{f(s)}{f\left(s_{0}\right)}\right|<e^{M}
$$

in $\left\{s:\left|s-s_{0}\right| \leq r\right\}$ with $M>1$, then

$$
\left|\frac{f\left(s_{0}\right)}{f(s)} \prod_{\rho} \frac{s-\rho}{s_{0}-\rho}\right|<e^{C M}
$$


for $\left|s-s_{0}\right| \leq \frac{3}{8} r$, where $C$ is some constant and $\rho$ runs through the zeros of $f(s)$ such that $\left|\rho-s_{0}\right| \leq \frac{1}{2} r$.

Proof. The lemma follows immediately from the proof of Lemma $\alpha$ in Titchmarsh [21, §3.9].

In order to apply Lemma 5, we need information about the growth of the Lerch zeta-function. For each $\sigma$, we define a number $\mu(\sigma)=$ $\mu(\lambda, \alpha, \sigma)$ as the lower bound of numbers $\xi$ such that $L(\lambda, \alpha, \sigma+i T) \ll$ $T^{\xi}$.

Lemma 6. Let $0<\lambda, \alpha \leq 1$ and $\sigma_{0}<0$. Then

$$
\mu(\sigma) \leq \begin{cases}\frac{1}{2}-\sigma & \text { if } \sigma_{0} \leq \sigma \leq 0, \\ \frac{1}{2}+\left(\frac{64}{205}-1\right) \sigma & \text { if } 0 \leq \sigma \leq \frac{1}{2}, \\ \frac{64}{205}(1-\sigma) & \text { if } \frac{1}{2} \leq \sigma \leq 1, \\ 0 & \text { if } \sigma \geq 1\end{cases}
$$

Proof. In [5], it is proved that

$$
L\left(\lambda, \alpha, \frac{1}{2}+i t\right) \ll t^{\frac{32}{205}+\varepsilon} \quad(t \rightarrow \infty),
$$

and from the approximation of the Lerch zeta-function by a finite sum ([14, Theorem 1.2 in Chapter 3]) we see that $L(\lambda, \alpha, \sigma+i t) \ll t^{\varepsilon}$ for $\sigma \geq 1$. Now the lemma follows by the Phragmén-Lindelöf theorem (see Titchmarsh [20, §5.65]) and by the functional equation (2) in view of Stirling's formula (see Titchmarsh [20, §4.42])

$$
|\Gamma(1-s)|=\sqrt{2 \pi}|t|^{\frac{1}{2}-\sigma} e^{-\frac{\pi|t|}{2}}\left(1+O\left(|t|^{-1}\right)\right) \quad(|t| \rightarrow \infty),
$$

uniformly for $\sigma_{0}<\sigma \leq 1 / 2$.

Proof of Proposition 2. To prove the proposition we choose $f(s)=$ $L(\lambda, \alpha, s), s_{0}=3+i t$, and a sufficiently large but fixed radius $r$ in Lemma 5. In view of Lemma 6 we take $M=b \log T$, where $b=b(r)$. The function $1 / L\left(\lambda, \alpha, s_{0}\right)$ is bounded. By the formula (4) for a number of nontrivial zeros, we have that the number of zeros in the disc $\left|s-s_{0}\right|<\frac{1}{2} r$ is $<c \log \Im s_{0}$. This proves Proposition 2 .

\section{Proof of Theorem 3}

Proof of Theorem [3. If $\lambda=1 / 2,1$, then in view of equalities (11) it is well known that the non-real complex number $\rho$ is a zero of $L(\lambda, \lambda, s)$ if and only if $1-\bar{\rho}$ is also a zero of $L(\lambda, \lambda, s)$.

Next we assume that $0<\lambda<1$ and $\lambda \neq 1 / 2$. By the formula (4), we see that the number of zeros in the disc $|s-\rho|<\exp \left(-A \gamma / \log ^{2} \gamma\right)$ 
is $<c \log \Im \rho$. Let $r_{k}=k \exp (-A \gamma / \log \gamma), k=1, \ldots,[c \log \gamma]+1$. By Dirichlet's box principle, there is $1 \leq \ell \leq[c \log \gamma]$ such that $L(\lambda, \lambda, s)$ has no zeros for the ring

$$
r_{\ell}<|s-\rho| \leq r_{\ell+1} .
$$

Let

$$
r=\left(r_{\ell}+r_{\ell+1}\right) / 2=(\ell+1 / 2) \exp (-A \gamma / \log \gamma) .
$$

Suppose $G(s)$ and $P(s)$ are defined by the functional equation (3). Let $C_{R}=\{s:|s-\rho|=r\}$ and $C_{L}=\{s:|s-(1-\bar{\rho})|=r\}$. The steps of the proof are the following. If $L(\lambda, \lambda, s)$ has $N$ zeros inside of $C_{R}$, then by Rouché's theorem we expect that $G(s) L(\lambda, \lambda, s)+P(s)$ has $N$ zeros inside $C_{R}$. Then by the functional equation (3), the function $L(1-\lambda, \lambda, 1-s)$ has $N$ zeros inside $C_{R}$, then by conjugation $L(\lambda, \lambda, s)$ has $N$ zeros inside $C_{L}$. Next we need to justify the step involving Rouché's theorem.

Note that $G(s)$ has no zeros. By Rouché's theorem, the functions $G(s) L(\lambda, \lambda, s)$ and $G(s) L(\lambda, \lambda, s)+P(s)$ have the same number of zeros inside of the circle $C_{r}$ if on this circle the inequality

$$
|P(s)|<|G(s) L(\lambda, \lambda, s)|
$$

is valid.

In view of the growth of the Lerch zeta-function (see Lemma 6) we get that, for sufficiently large $t$ and $-1.4 \leq \sigma \leq 2$,

$$
|P(s)|<|\Gamma(s)| t^{2} e^{-\pi t / 2} \text { and }|G(s)| \geq(2 \pi)^{-2}|\Gamma(s)| e^{\pi t / 2} .
$$

Proposition 2 gives, for $s \in C_{R}$,

$$
L(\lambda, \lambda, s) \gg \exp (-(A C+o(1)) \gamma)
$$

where $A C<\pi$. Thus the inequality (8) is valid. By this Theorem 3 is proved.

Note, that from this proof we have that the quantity $\log ^{2} \gamma$ in the inequality (3) of Theorem 3 can be replaced (at the expense of more complicated notations) by the smaller quantity $c \log \gamma+1$, where $c$ is from the proof of Theorem 3 .

\section{REFERENCES}

[1] T. M. Apostol, On the Lerch zeta function, Pacific J. Math. 1 (1951), 161167. Addendum, Pacific J. Math. 2(1952), 10.

[2] E. P. Balanzario, J. Sanchez-Ortiz, Zeros of the Davenport-Heilbronn counterexample, Math. Comput. 76 (2007), 2045-2049. 
[3] B. C. Berndt, Two new proofs of Lerch's functional equation, Proc. Amer. Math. Soc. 32 (1972), 403-408.

[4] E. Bombieri, D. A. Hejhal, On the distribution of zeros of linear combinations of Euler products, Duke Math. J. 80 (1995), 821-862.

[5] R. Garunkštis, Growth of the Lerch zeta-function, Lith. Math. J. 45 (2005), $34-43$.

[6] R. Garunkštis, A. LaurinčIKas, On zeros of the Lerch zeta-function, Number theory and its applications, S. Kanemitsu and K. Györy (editors), Kluwer Academic Publishers 1999, 129-143.

[7] R. Garunkštis, R. A. Laurinčikas, J. Steuding, On the mean square of Lerch zeta-functions, Arch. Math. 80 (2003), 47-60.

[8] R. Garunkštis, J. Steuding, On the zero distributions of Lerch zetafunctions, Analysis 22 (2002), 1-12.

[9] R. Garunkštis, J. Steuding, Do Lerch zeta-functions satisfy the Lindelof hypothesis?, in: Analytic and Probabilistic Methods in Number Theory, Proceedings of the Third Intern. Conf. in Honour of J. Kubilius, Palanga, Lithuania, 24-28 September 2001, (eds. A. Dubickas, A. Laurinčikas and E. Manstavičius), TEV, Vilnius, (2002), 61-74.

[10] R. Garunkštis, R. ŠImĖnas, On the Speiser equivalent for the Riemann hypothesis, Eur. J. Math. 1 (2015), 337-350.

[11] J. C. Lagarias, W.-C. W. LI, The Lerch zeta function I. Zeta integrals, Forum Math. 24 (2012), 1-48.

[12] J. C. Lagarias, W.-C. W. Li, The Lerch zeta function II. Analytic continuation, Forum Math. 24 (2012), 49-84.

[13] A. LaURINČIKAS, Universality of the Lerch zeta function, Lithuanian Math. J. 37 (1997), 275-280.

[14] A. LaurinČIKas, R. Garunkštis, The Lerch zeta-function, Kluwer Academic Publishers, 2002, 197 pp.

[15] Y. Lee, T. Nakamura, Ł. Pańkowski, Joint universality for Lerch zetafunctions, J. Math. Soc. Japan 69 (2017), 153-161.

[16] M. LeRCH, Note sur la fonction $\mathcal{K}(z, x, s)=\sum_{k=0}^{\infty} e^{2 k \pi i x}(z+k)^{-s}$, Acta Math. 11 (1887), 19-24.

[17] M. Mikolás, New proof and extension of the functional equality of Lerch's zeta-function, Ann. Univ. Sci. Budapest. Sect. Math. 14 (1971), 111-116.

[18] F. Oberhettinger, Note on the Lerch zeta-function, Pacific J. Math. 6 (1956), 117-120.

[19] R. SpIRA, Zeros of Hurwitz zeta-functions, Math Comput. 136 (1976), 863866.

[20] E. C. Titchmarsh, The theory of functions, 2nd ed., Oxford University Press, 1939

[21] E. C. Titchmarsh, The theory of the Riemann zeta-function. 2nd ed., rev. by D. R. Heath-Brown, Oxford Science Publications. Oxford: Clarendon Press, 1986.

[22] R. C. Vaughan, Zeros of Dirichlet series, Indagationes Mathematicae 26 (2015), 897-909. 
Ramūnas GarunKštis, Department of Mathematics and Informatics, Vilnius University, Naugarduko 24, 03225 Vilnius, Lithuania

E-mail address: ramunas.garunkstis@mif.vu.lt

$U R L$ : www.mif . vu.1t/ garunkstis

Rokas TAMošiūnas, Department OF MAThematics AND InFormatics, Vilnius University, Naugarduko 24, 03225 Vilnius, Lithuania

E-mail address: rokas.tamosiunas@mif.vu.lt 